\title{
Malaria congénita por Plasmodium falciparum
}

\section{Congenital malaria by Plasmodium falciparum}

\author{
Del Castillo Calderón, José Gabriel ${ }^{a}$, Angie Milena Cárdenas Silva ${ }^{b}$
}

aMédico Pediatra. Grupo de investigación. Hospital Infantil Los Ángeles. Colombia

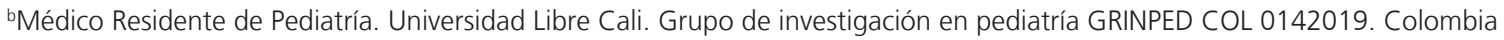

Recibido: 4 de junio de 2019; Aceptado: 5 de febrero de 2020

\section{¿Qué se sabe del tema que trata este estudio?}

La malaria congénita es la infección por Plasmodium spp transmitida desde la madre al feto durante la gestación o intraparto. Esta infeccion aumenta el riesgo de retardo de crecimiento intrauterino y parto pretérmino y puede causar enfermedad grave en el recién nacido.

\section{¿Qué aporta este estudio a lo ya conocido?}

Este artículo resalta considerar la malaria congénita como diagnóstico diferencial de sepsis neonatal en hijos de mujeres residentes en áreas no endémicas y que migran o visitan áreas endémicas para malaria.

\section{Resumen}

La malaria congénita (MC) es la infección por Plasmodium spp adquirida in útero o durante el parto y sus manifestaciones clínicas son inespecíficas. Puede causar enfermedad grave en la embarazada y en el recién nacido. Objetivo: describir dos casos de MC causados por Plasmodium falciparum, diagnóstico diferencial de sepsis en recién nacidos de gestantes que hayan visitado o residan en áreas endémicas para malaria. Casos Clínicos: Neonatos de sexo femenino, nacidos en área no endémica para malaria, diagnosticados con sepsis neonatal y tratados con antibióticos sin respuesta clínica. Después de la primera semana de vida la gota gruesa identificó trofozoítos de Plasmodium falciparum y los neonatos recibieron tratamiento con quinina intravenosa con mejoría. Las madres de las recién nacidas tuvieron malaria en el embarazo, una de ellas recibió tratamiento y estaba asintomática y otra tenía malaria complicada al momento del parto. Conclusiones: La MC puede causar enfermedad neonatal grave con manifestaciones clínicas inespecíficas y similares a la sepsis, el tratamiento oportuno disminuye el riesgo de malaria complicada. Es un diagnóstico diferencial en recién nacidos de mujeres con malaria durante el embarazo o gestantes que visiten o residan en áreas endémicas.
Palabras clave:

Malaria;

Plasmodium falciparum; neonato; congénita; sepsis 


\section{Abstract}

Congenital malaria (CM) is a Plasmodium spp infection acquired in utero or during delivery with nonspecific clinical manifestations. Plasmodium falciparum can cause severe illness in pregnant women and newborns. Objective: to describe two cases of CM caused by Plasmodium falciparum, differential diagnosis of sepsis in newborns of pregnant women who live in or have visited endemic malaria zones. Clinical Cases: Female neonates born in a non-endemic malaria area, diagnosed with neonatal sepsis and treated with antibiotics without clinical response. After the first week of life, the peripheral blood smear identified trophozoites of Plasmodium falciparum thus the newborns were treated with intravenous quinine, improving their condition. The mothers of the two newborns who had malaria in pregnancy, one of them received treatment and she was asymptomatic, and the other one had severe malaria at the time of delivery. Conclusions: CM can cause severe neonatal disease with non-specific, sepsis-like clinical manifestations in which early treatment decreases the risk of complicated malaria. It is a differential diagnosis in newborns of women with a history of malaria during pregnancy or pregnant women visiting or living in endemic malaria areas.
Keywords:

Malaria;

Plasmodium falciparum;

newborn;

congenital;

sepsis

\section{Introducción}

En Colombia en 2019 se notificaron 74.409 casos de malaria, de ellos 455 en gestantes $(0,6 \%)^{1}$. Desde 2015, en las Américas aumentaron los casos en Venezuela y los brotes en países que avanzaban hacia la eliminación como Costa Rica, República Dominicana y Ecuador e incrementó la transmisión en áreas endémicas (Brasil, Colombia, Guyana, Nicaragua y Panamá). Paraguay y Argentina recibieron la certificación de países libres de malaria en 2018 y 2019, respectivamente ${ }^{2}$. Plasmodium vivax causa el $74,1 \%$ de los casos ${ }^{3}$.

La malaria congénita (MC) ocurre por infección in útero o durante el parto y se define por la identificación de formas asexuales del parásito en sangre periférica del neonato entre las primeras $24 \mathrm{~h}$ y los siguientes siete días de vida ${ }^{4}$, la incidencia es variable en áreas endémicas y no endémicas, reportándose entre 0,3 a $37 \% 5$. Los signos y síntomas son inespecíficos, el diagnóstico requiere un índice de sospecha, examen físico cuidadoso y gotas gruesas secuenciales, puede confundirse con sepsis neonatal y STORCH (sífilis, toxoplasmosis, rubeola, citomegalovirus, herpes virus) ${ }^{6}$.

La malaria complicada sin tratamiento es mortal, causa coma, acidosis metabólica, anemia grave, ictericia, hipoglicemia, falla renal aguda, edema pulmonar, coagulopatía, choque o hiperparasitemia ${ }^{7}$.

La gota gruesa identifica trofozoítos de Plasmodium spp y consiste en obtener una gota de sangre que se dispone en una lámina portaobjetos, es teñida con colorantes derivados de Romanowsky y observada al microscopio de luz ${ }^{8}$. El estándar de oro para el diagnóstico de $\mathrm{MC}$ es la identificación del parásito en sangre periférica neonatal ${ }^{9}$, la presencia de parásitos en sangre de cordón puede no representar infección activa ${ }^{10}$.

Se encontraron menos de 20 casos de MC reporta- dos en Colombia en la literatura revisada y uno causado por Plasmodium falciparum (P. falciparum) ${ }^{11}$. Este trabajo tiene como objetivo describir dos casos de MC causados por esta especie de Plasmodium, diagnóstico diferencial en recién nacidos de gestantes que visiten o residan en áreas endémicas para malaria.

\section{Caso Clínico}

\section{Caso 1}

Recién nacido femenino de 25 días de vida consultó por dos días de ictericia, fiebre, irritabilidad e hiporexia. Hija de primigestante de 20 años, nació en área no endémica para malaria (Pasto ubicada a $2.527 \mathrm{~m}$ s.n.m) a las 38 semanas de gestación vía cesárea por oligohidramnios y bajo peso al nacer (2.115 g). Ella estaba pálida, ictérica y febril, tenía petequias y hepatoesplenomegalia. Se diagnosticó sepsis neonatal y sospecha de infección STORCH y fue tratada con antibióticos. El hemograma mostró leucopenia $\left(3.600 \mathrm{~mm}^{3}\right)$ y trombocitopenia $\left(66.000 / \mathrm{mm}^{3}\right)$, sin anemia $(11 \mathrm{~g} / \mathrm{dL})$; reticulocitosis (6\%); proteína C reactiva elevada $(18 \mathrm{mg} /$ $\mathrm{dl}$ ); hiperbilirrubinemia (bilirrubina total $13,9 \mathrm{mg} / \mathrm{dl}$, directa $0,9 \mathrm{mg} / \mathrm{dl}$ ); glucosa por punción normal. Los cultivos fueron negativos al igual que los estudios de inmunoglobulina $\mathrm{M}$ para TORCH, prueba no treponémica y virus de inmunodeficiencia humana (VIH). Posteriormente, la madre informó que viajó zona endémica para malaria (Urabá) a las 22 semanas de gestación y tuvo infección por P. falciparum tratada con quinina, estaba asintomática al momento del parto. La prueba rápida de anticuerpos del neonato fue positiva para $P$. falciparum y la gota gruesa identificó 5.400 trofozoítos/ $\mu \mathrm{L}$ (figura 1), se inició quinina intravenosa (IV) $10 \mathrm{mg} / \mathrm{kg}$ cada $8 \mathrm{~h}$ por 3 días. Las gotas gruesas de control (48 h, 72 h y 7 días) fueron negativas. Egresó 


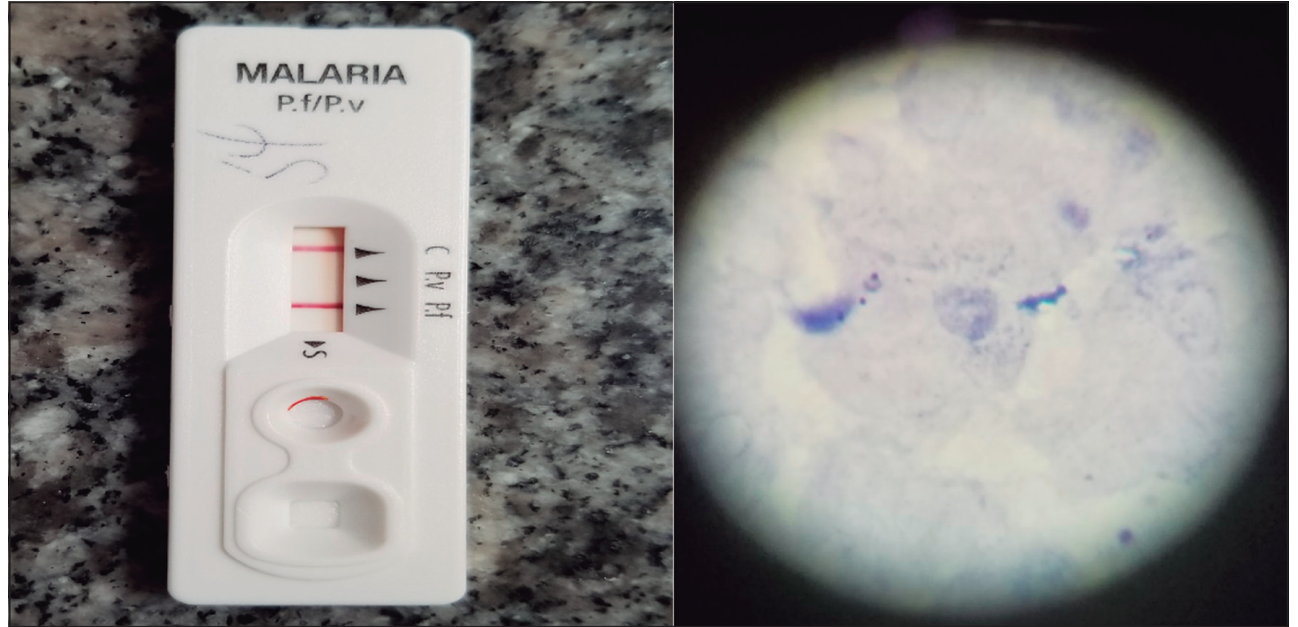

Figura 1. A. Test rápido de anticuerpos para infección por Plasmodium. B. Extendido de sangre periférica con evidencia de trofozoitos de $P$. falciparum. sin complicaciones y estaba asintomática a los 8 días posterior al alta.

\section{Caso 2}

Neonato de sexo femenino, hija de primigestante de 18 años con malaria complicada y sepsis obstétrica al momento del parto, nació a las 36 semanas de gestación en zona no endémica para malaria (Pasto), vía cesárea de emergencia por estado fetal no satisfactorio y oligohidramnios grave. Tuvo bajo peso de nacimiento $(2.410 \mathrm{~g})$, estaba impregnada de meconio, hipotónica, hipotérmica y cianótica, ingresó a cuidado intensivo neonatal por dificultad respiratoria y sepsis. Se inició tratamiento antibiótico, el hemograma documentó leucocitosis $\left(26.000 / \mathrm{mm}^{3}\right)$, sin anemia o trombocitopenia (hemoglobina $14.4 \mathrm{~g} /$ dl y plaquetas $190.000 / \mathrm{mm}^{3}$ ), glucosa por punción 47 $\mathrm{mg} / \mathrm{dl}$. La prueba de VIH y los cultivos fueron negativos. Continuó dependiente de oxígeno al séptimo día de vida, el hemograma evidenció pancitopenia (leucocitos $4.800 / \mathrm{mm}^{3}$, hemoglobina $8,3 \mathrm{~g} / \mathrm{dl}$, plaquetas $13.000 / \mathrm{mm}^{3}$ ) y la gota gruesa identificó 83 trofozoítos $/ \mu \mathrm{L}$ de $P$. falciparum. Recibió quinina IV dosis inicial $20 \mathrm{mg} / \mathrm{kg}$ seguida de $10 \mathrm{mg} / \mathrm{kg} /$ dosis cada $8 \mathrm{~h}$ por 3 días con mejoría y controles de gota gruesa negativos ( 48 h, 72 h y 7 días). Egresó del hospital sin complicaciones.

\section{Discusión}

Se describen dos neonatos con MC por P. falciparum, nacidos en área no endémica para malaria y afectados por oligohidramnios y bajo peso de nacimiento, se hizo diagnóstico con gota gruesa de sangre periférica y se usó quinina IV para el tratamiento. Madres primi- gestantes, una de ellas estaba asintomática y otra con malaria complicada al momento del parto. Las áreas de transmisión de malaria están debajo de 1.700 m s.n.m en Colombia, los neonatos nacieron y permanecieron en Pasto ubicada a 2.527 m s.n.m lo cual descarta la infección neonatal.

La malaria puede causar enfermedad grave en embarazadas con ninguna o poca inmunidad previa y mujeres de áreas no endémicas viajeras a zonas endémicas ${ }^{10}$. En áreas de alta transmisión las primigestantes producen anticuerpos y están parcialmente protegidas en embarazos posteriores, lo cual no ocurre en áreas de baja transmisión ${ }^{12}$.

$P$. falciparum invade eritrocitos de todas las edades, tiene parasitemias elevadas ${ }^{13}$, hay secuestro placentario de eritrocitos parasitados por la interacción del condroitín sulfato A y la proteína VAR2CSA (Variant Surface antigen 2-CSA) ${ }^{14}$, esto activa la inflamación, la migración de monocitos y la liberación de factores humorales que podrían favorecer el parto pretérmino.

El engrosamiento de la placenta, la reducción del transporte transplacentario de nutrientes y de la producción de hormonas, alteración en la angiogénesis, cambios en la arquitectura vellosa y en el flujo sanguíneo útero placentario deterioran el crecimiento fe$\operatorname{tal}^{12,13}$.

El riesgo de transmisión vertical es 1 a $4 \%{ }^{13}$, el paso transplacentario de anticuerpos IgG, la hemoglobina fetal y las bajas presiones parciales de oxígeno en la circulación fetal son mecanismos de defensa ${ }^{15}$.

La Organización Mundial de la Salud (OMS) recomienda quinina y clindamicina para tratamiento de malaria no complicada por P. falciparum en el primer trimestre del embarazo y terapia combinada basada en artemisina en trimestres posteriores ${ }^{7}$. En el caso 1 la gestante recibió quinina a las 22 semanas de gestación, 
la cual ha mostrado menor eficacia que los derivados de la artemisina ${ }^{16} \mathrm{y}$ aunque cruza la placenta no es terapéutico para el feto ${ }^{17}$.

Los síntomas y signos inespecíficos en el caso 1 pueden atribuirse a la infección tardía durante el embarazo y la necesidad de varios ciclos eritrocíticos para causar enfermedad ${ }^{10}$. Las manifestaciones clínicas ocurren entre los 10 y 28 días posnatales, $80 \%$ de los pacientes tienen fiebre, anemia y esplenomegalia y un tercio presentan ictericia e hiperbilirrubinemia ${ }^{18}$.

En el caso 2 la infección ocurrió en el último trimestre del embarazo, la gestante tuvo malaria complicada y el neonato nació pretérmino, con bajo peso de nacimiento, dificultad respiratoria e hipoglicemia. En la circulación fetal ocurre ciclo el eritrocitario de replicación asexual de $P$. falciparum liberando merozoitos cada $48 \mathrm{~h}^{19}$, la sintomatología puede ser leve, tardía o ausente debido a que los anticuerpos antimaláricos evitan o atenúan la replicación del parásito ${ }^{10}$.

Para el tratamiento de malaria no complicada por $P$. falciparum en niños $<5 \mathrm{~kg}$ la terapia combinada basada en artemisina con dosis iguales que los niños $>5 \mathrm{~kg}$ es la recomendación de OMS. Artesunato, artemeter o quinina tienen indicación para tratamiento de malaria complicada ${ }^{7}$.

Las guías nacionales recomiendan artemeter más lumefantrina para tratamiento en niños $>5 \mathrm{~kg}$ y quinina y clindamicina en niños $<5 \mathrm{~kg}$, pero en neonatos sugieren no usar clindamicina ${ }^{20,21}$; Artemeter está disponible en comprimidos y Artesunato no estaba disponible en el país en el momento de la presentación de los casos. Los neonatos se trataron según las guías locales, el caso 2 se clasificó como malaria complicada por lo cual se administró dosis de carga de quinina.

El pronóstico depende de la especie de Plasmodium, la región, la inmunidad de la embarazada y la parasitemia ${ }^{9}$.

\section{Conclusión}

La MC puede causar enfermedad neonatal grave con manifestaciones clínicas inespecíficas y similares a la sepsis, el tratamiento oportuno disminuye el riesgo de malaria complicada. Es un diagnóstico diferencial en recién nacidos de mujeres con malaria durante el embarazo o gestantes que visiten o residan en áreas endémicas.

\section{Responsabilidades Éticas}

Protección de personas y animales: Los autores declaran que los procedimientos seguidos se conformaron a las normas éticas del comité de experimentación humana responsable y de acuerdo con la Asociación Médica Mundial y la Declaración de Helsinki.

Confidencialidad de los datos: Los autores declaran que han seguido los protocolos de su centro de trabajo sobre la publicación de datos de pacientes.

Derecho a la privacidad y consentimiento informado: Los autores han obtenido el consentimiento informado de los pacientes y/o sujetos referidos en el artículo. Este documento obra en poder del autor de correspondencia.

\section{Conflicto de intereses}

Los autores declaran no tener conflicto de intereses.

\section{Agradecimientos}

A la Fundación Hospital Infantil Los Ángeles y la Oficina de Educación Médica por fomentar la investigación en su centro.

\section{Referencias}

1. Instituto Nacional de Salud - INS. Informe evento Malaria a periodo epidemiológico XII-2019. Colombia. Disponible en: https://www.ins.gov.co/ buscador-eventos/Informesdeevento/ MALARIA\%20PE\%20XII\%202019.pdf. Ultimo acceso 31 de enero 2020.

2. Organización Panamericana de la Salud/Organización Mundial de la salud. Actualización Epidemiológica: Malaria en las Américas. Noviembre 18 de 2019. Disponible en: https://www. paho.org/hq/index.php?option=com docman\&view= download\&category slug $=2019$ - 3 \&alias $=51011-18$ de-noviembre-de-2019-malaria-
actualizacion-epidemiologica\&Itemid $=2$ 70\&lang=es. Ultimo acceso 31 de enero 2020.

3. Organización Mundial de la Salud OMS. Paludismo: datos y cifras. Disponible en: https://www.who.int/es/news-room/factsheets/detail/malaria. Ultimo acceso 31 de enero de 2020.

4. Bhatia R, Rajwaniya D, Agrawal P. Congenital Malaria due to Plasmodium vivax Infection in a Neonate. Case Rep Pediatr. 2016; 1-2.

5. Romani L, Pane S, Severini C, Menegon M, Foglietta G, Bernardi S, et al. Challenging diagnosis of congenital malaria in non-endemic areas. Malar J. 2018; 17(1):470.

6. Uneke CJ. Congenital Plasmodium falciparum malaria in sub-Saharan Africa: a rarity or frequent occurrence? Parasitol Res. 2007;101(4):835-42. DOI 10.1007/ s00436-007-0577-9.

7. World Health Organization. Guidelines for the treatment of malaria. 3rd Edition. Geneva. 2015. Disponible en: https://apps.who.int/iris/bitstream/ handle/10665/162441/9789241549127_ eng.pdf;jsessionid $=458$ FA9FC732EB76 C5CD24DF3C4C3D99B? sequence $=1$. Ultimo acceso 31 de enero de 2020.

8. Instituto Nacional de Salud - Grupo de Parasitología. Guía para la vigilancia por laboratorio de parásitos del género Plasmodium spp. Colombia, Bogotá. 2017. Pág. 9. Disponible en: https:// www.ins.gov.co/buscador-eventos/ 
Informacin $\% 20 \mathrm{de} \% 20$ laboratorio/ Gu\%C3\%ADa\%20Vigilancia\%20por\%20 laboratorio\%20Plasmodium\%20spp.pdf. Ultimo acceso 31 de enero de 2020.

9. Gulasi S, Özdener N. Congenital malaria: Importance of diagnosis and treatment in pregnancy. Turk J Pediatr 2016;58:195-9.

10. Michaels M, Sánchez P, Ling L.

Toxoplasmosis, sífilis, paludismo y tuberculosis congénitos. En: Gleason \& Juul. Avery. Enfermedades del recién nacido. España: Elsevier, 2019; 527-52.

11. Piñeros-Jiménez JG, Álvarez G, Tobón A, Arboleda M, Carrero S, Blair S. Congenital malaria in Urabá, Colombia. Malar J. 2011;10:239.

12. Bauserman M, Conroy AL, North K, Patterson J, Bose C, Meshnick S. An overview of malaria in pregnancy. Semin Perinatol 2019;43(5):282-90.

13. Wylie BJ, Rogerson S. Malaria in pregnancy: Epidemiology, clinical manifestations, diagnosis, and outcome. [Internet]. UpToDate; 2019. Disponible en: http://www.uptodate.com/. Último acceso: 27 de octubre de 2019.
14. Seitz J, Morales-Prieto DM, Favaro RR, Schneider H, Markert UR. Molecular Principles of Intrauterine Growth Restriction in Plasmodium Falciparum Infection. Front Endocrinol. 2019;10:118.

15. Menendez C, Mayor A. Congenital malaria: The least known consequence of malaria in pregnancy. Semin Fetal Neonatal Med 2007;12:207-13.

16. Saito M, Gilder M, McGready R, Nosten F. Antimalarial drugs for treating and preventing malaria in pregnant and lactating women, Expert Opin Drug Saf. 17(11):1129-44.

17. Quinine. In: Lexi-drugs online [database on the Internet]. Hudson $(\mathrm{OH})$ : Wolters Kluwer Clinical Drug Information, Inc.: 2019 [cited 29 September 2019]. Disponible en: https://www-uptodatecom.sibulgem.unilibre.edu.co/contents/ quinine-drug-information?search= QUININA\&source=panel_search_ result\&selectedTitle $=1 \sim 95 \&$ usage type $=$ panel\&kp_tab $=$ drug general\&display_rank=1\#F3343867.
Subscription required to view.

18. Maldonado YA. Malaria and Less Common Protozoan and Helminth Infections. Remington and Klein's Infectious Diseases of the Fetus and Newborn Infant. En: Wilson C, Nizet V, Maldonado Y, Remington J, Klein J. Remington and Klein's Infectious Diseases of the Fetus and Newborn Infant. Philadelphia: Elsevier, 2016; 1043-57.

19. Vásquez Ana María, Tobón Alberto. Mecanismos de patogenia en la malaria por Plasmodium falciparum. Biomédica. 2012;32(Suppl 1):106-20

20. Ministerio de Salud y Protección Social-Federación Médica Colombiana. Malaria - memorias. Colombia Bogotá. 2012-2013. Disponible en: https://www.minsalud.gov.co/sites/rid/ Lists/BibliotecaDigital/RIDE/VS/TH/ memorias_malaria.pdf. Ultimo acceso 31 de enero de 2020.

21. Padilla Julio C, Montoya Roberto. Guía de Atención Clínica de Malaria: Guideline for care clinics for malaria. Infect. 2011;15(4):302-23. 